myeloma and associated with relapse and shorter survival. Leukemia. 2005;19:1248-52.

6. Caenepeel S, Brown SP, Belmontes B, Moody G, Keegan KS, Chui D, et al. AMG 176, a selective MCL1 inhibitor, is effective in hematologic cancer models alone and in combination with established therapies. Cancer Discov. 2018;8:1582-97.

7. Leverson JD, Zhang H, Chen J, Tahir SK, Phillips DC, Xue J, et al. Potent and selective small-molecule MCL-1 inhibitors demonstrate on-target cancer cell killing activity as single agents and in combination with ABT-263 (navitoclax). Cell Death Dis. 2015;6:e1590.

8. Kotschy A, Szlavik Z, Murray J, Davidson J, Maragno AL, Le Toumelin-Braizat G, et al. The MCL1 inhibitor S63845 is tolerable and effective in diverse cancer models. Nature. 2016;538: 477-82.

9. Tron AE, Belmonte MA, Adam A, Aquila BM, Boise LH, Chiarparin E, et al. Discovery of Mcl-1-specific inhibitor AZD5991 and preclinical activity in multiple myeloma and acute myeloid leukemia. Nat Commun. 2018;9:5341.

10. Hideshima T, Bergsagel PL, Kuehl WM, Anderson KC. Advances in biology of multiple myeloma: clinical applications. Blood. 2004;104:607-18.

11. Jourdan M, Veyrune JL, De Vos J, Redal N, Couderc G, Klein B. A major role for Mcl-1 antiapoptotic protein in the IL-6-induced survival of human myeloma cells. Oncogene. 2003;22:2950-9.
12. Jourdan M, De Vos J, Mechti N, Klein B. Regulation of Bcl-2family proteins in myeloma cells by three myeloma survival factors: interleukin-6, interferon-alpha and insulin-like growth factor 1. Cell Death Differ. 2000;7:1244-52.

13. Puthier D, Derenne S, Barille S, Moreau P, Harousseau JL, Bataille R, et al. Mcl-1 and Bcl-xL are co-regulated by IL- 6 in human myeloma cells. Br J Haematol. 1999;107:392-5.

14. Luedtke DA, Niu X, Pan Y, Zhao J, Liu S, Edwards H, et al. Inhibition of Mcl-1 enhances cell death induced by the Bcl-2selective inhibitor ABT-199 in acute myeloid leukemia cells. Signal Transduct Target Ther. 2017;2:17012.

15. Niu X, Zhao J, Ma J, Xie C, Edwards H, Wang G, et al. Binding of released Bim to Mcl-1 is a mechanism of intrinsic resistance to ABT-199 which can be overcome by combination with daunorubicin or cytarabine in AML cells. Clin Cancer Res. 2016;22:4440-51.

16. Souers AJ, Leverson JD, Boghaert ER, Ackler SL, Catron ND, Chen J, et al. ABT-199, a potent and selective BCL-2 inhibitor, achieves antitumor activity while sparing platelets. Nat Med. 2013;19:202-8.

17. Matulis SM, Gupta VA, Nooka AK, Hollen HV, Kaufman JL, Lonial S, et al. Dexamethasone treatment promotes Bcl-2 dependence in multiple myeloma resulting in sensitivity to venetoclax. Leukemia. 2016;30:1086-93.

Leukemia (2019) 33:2102-2104

https://doi.org/10.1038/s41375-019-0437-5

Acute lymphoblastic leukemia

\title{
Anti-CD19 CAR-T as a feasible and safe treatment against central nervous system leukemia after intrathecal chemotherapy in adults with relapsed or refractory B-ALL
}

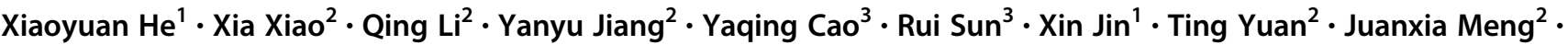 \\ $\mathrm{Li} \mathrm{Ma}{ }^{2} \cdot$ Wenyi $\mathrm{Lu}^{2} \cdot$ Cuicui $\mathrm{Lyu}^{2} \cdot$ Kaiqi Liu ${ }^{4} \cdot$ Mingfeng $\mathrm{Zhao}^{2,1}$
}

Received: 29 November 2018 / Revised: 14 February 2019 / Accepted: 15 February 2019 / Published online: 7 March 2019

(c) The Author(s) 2019. This article is published with open access

These authors contributed equally: Mingfeng Zhao, Kaiqi Liu

Supplementary information The online version of this article (https:// doi.org/10.1038/s41375-019-0437-5) contains supplementary material, which is available to authorized users.

Kaiqi Liu

yanghua94583@sina.com

$\triangle$ Mingfeng Zhao

mingfengzhao@sina.com

1 Nankai University School of Medicine, Tianjin, PR China

\section{To the Editor:}

Chimeric antigen receptor (CAR)- $\mathrm{T}$ cell treatment as an emerging tumor immunotherapy has produced exciting results in relapsed or refractory B cell acute lymphoblastic leukemia (B-ALL) [1-4]. However, few data are available on the therapeutic effect of CAR-T against central nervous system leukemia (CNSL). Here, we evaluated the feasibility and safety of

2 Department of hematology, Tianjin First Central Hospital, No. 24 Fu Kang Road, Tianjin, PR China

3 Tianjin medical university, Tianjin, PR China

4 Leukemia Center, Institute of Hematology and Blood Diseases Hospital, Chinese Academy of Medical Sciences and Peking Union Medical College, 288 Nanjing Road, Tianjin, PR China 
A

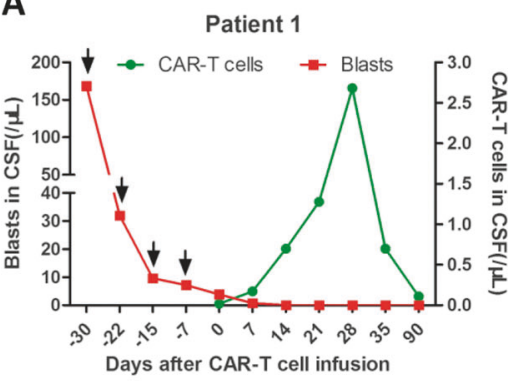

D
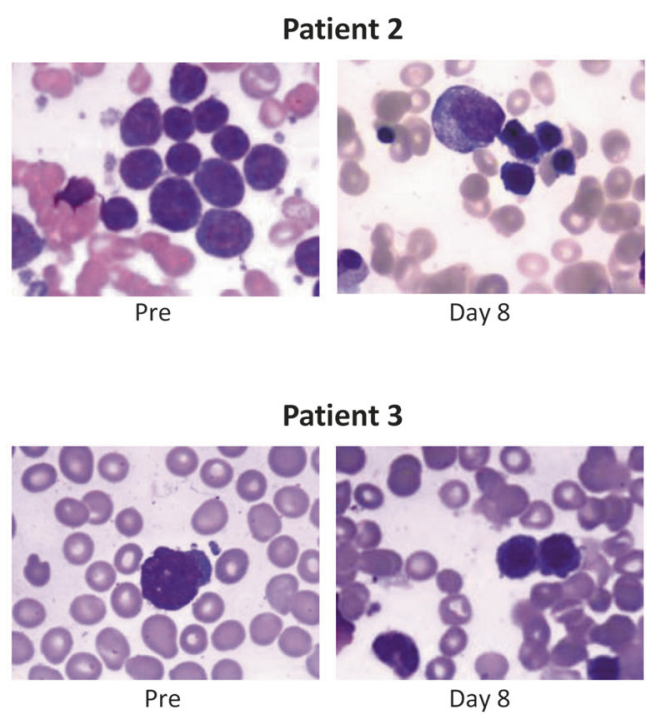

B

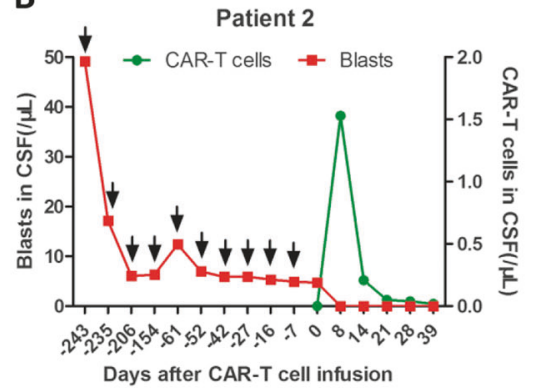

C

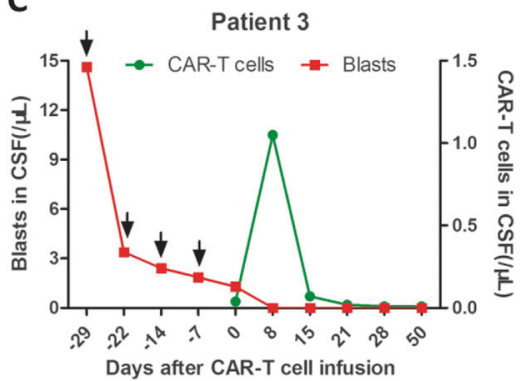

E

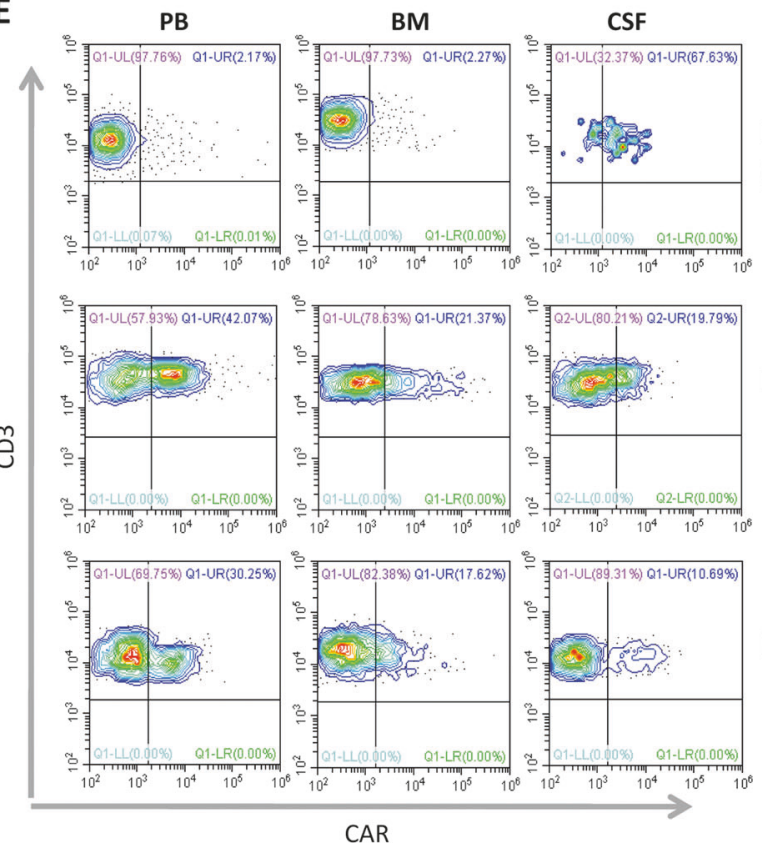

Patient 1

(Day 28)

Patient 2

(Day 8)

Patient 3

(Day 8)
Fig. 1 Clinical response, expansion and persistence of antiCD19chimeric antigen receptor (CAR)-T cells. a-c Cell counts of CAR-T cells and tumor cells in the cerebrospinal fluid (CSF) before and after CAR-T infusion. The black arrows indicate intrathecal

anti-CD19 CAR-T against CNSL after intrathecal chemotherapy in three adults with relapsed or refractory B-ALL.

Patient 1 with isolated CNSL, was refractory to high dose methotrexate plus vindesine and L-asparaginase, and intrathecal chemotherapy, accompanied by bone marrow (BM) sustained remission with minimal residual disease (MRD) negative. Patient 2 initially experienced a CNS relapse, and underwent intrathecal chemotherapy, systemic chemotherapy and radiotherapy. However, her CNSL was not controlled, accompanied by $\mathrm{BM}$ recurrence. Patient 3 received prophylactic intrathecal chemotherapy after his first complete remission (CR) but experienced a rapid recurrence in his BM and CNS. They were enrolled in our anti-CD19 CAR-T clinical trial (ChiCTR-ONN16009862). Prior to CAR-T cell infusion, all the patients received conditioning chemotherapy with fludarabine and cyclophosphamide, and intrathecal chemotherapy to reduce blasts in the cerebrospinal fluid (CSF). Detailed patient and chemotherapy. d Malignant cells in the bone marrow (BM) of patient 2 and patient 3 pre and post-infusion. e The peak expansion levels of CAR-T cells in the peripheral blood (PB), BM, and CSF

methodological information are described in Supplementary Methods, Table S1, and Figure S1.

First, we assessed the clinical response of CNSL to CAR-T therapy in these patients. All patients with CNSL achieved CR approximately one to two weeks post CAR-T infusion (Fig. 1a-c), accompanied by BM remission with MRD negative in patient 2 and patient 3 (Fig. 1d). One month after $\mathrm{CR}$, patient 2 received allogeneic hematopoietic stem cell transplantation. Until the most recent follow-up, her leukemia free survival has been over 2 months. Interestingly, patient 1 and patient 3 receiving no further therapy for CNSL after CAR-T infusion were in sustained remission for over 5 months (Table S1). A phase 1 dose-escalation trial reported that two B-ALL patients with CNSL achieved CR after CAR$\mathrm{T}$ therapy [3]. Another clinical trial showed that two CNSL patients at the time of CAR-T infusion subsequently had no blasts in the CSF [2]. Dai and colleagues also reported that two patients with active CNSL at the time of CAR-T infusion 
became CNS negative [5]. Altogether, CAR-T can be a feasible and effective treatment for CNSL.

We further evaluated the proliferation and persistence of CAR-T in vivo. Two patients reached the peak expansion of CAR-T cells in the CSF on day 8 (Fig. 1b, c), which coincided with the disappearance of CSF blasts in the responding patients. However, patient 1 exhibited peak expansion on day 28,2 weeks later than the disappearance of the tumor cells in the CSF (Fig. 1a). The persistence time of CAR-T cells in the CSF of patient 2 and patient 3 was about $2-3$ weeks (Fig. 1b, c), while in patient 1, 5.19\% of CAR-T cells persisted in the CSF on day 90 (Fig. 1a). Patient 1 who only had CNS relapse, showed significantly higher peak proportions of CAR-T cells in the CSF than those in the peripheral blood (PB) and BM. However, patient 2 and patient 3 both with BM and CNS recurrence exhibited markedly lower peak levels of CAR-T cells in the CSF compared to patient 1 (Fig. 1e), but relatively high peak levels in the PB and BM. These different distributions of CAR-T cells may be explained by chemotaxis and stimulated proliferation of effector cells at the tumor sites.

We next evaluated the adverse events associated with antiCD19 CAR-T treatment. Patient 1 only showed grade 1 anemia and grade 4 lymphopenia on day 3 after infusion (Table S2). She didn't complain of any discomfort post CAR$\mathrm{T}$ therapy. It was reported that the severity of the cytokine release syndrome (CRS) was correlated with tumor burdens and $\mathrm{T}$ cell proliferation $[2,3,6]$. However, the peak expansion time of patient 1 was two weeks later than the disappearance of the tumor cells in the CSF (Fig. 1a), which may explain the low risk of CRS. Patient 2 and patient 3 experienced grade 2 fever, grade 3 febrile neutropenia, grade 2 CRS, and grade 1 reduced consciousness. Patient 2 also had grade 1 cognitive impairment and grade 2 convulsion. Dexamethasone was administrated at $10 \mathrm{mg}$ q $8 \mathrm{~h}$ to control her seizure on day 8 , and was de-escalated on day 9 and discontinued on day 10 . These adverse events were well managed with supportive care and dexamethasone. Other adverse events related to CAR-T therapy are shown in Figure S2 and Table S2.

In summary, this study showed that anti-CD19 CAR-T could effectively eliminated leukemia cells in the CNS with fully reversible toxicity. We also found that patient with only CNS recurrence had higher levels of CAR-T in the CSF and relatively lower severity of toxic effects than those with BM and CNS recurrence. This study shows that anti-CD19 CAR-T might be a feasible and safe treatment for CNSL after intrathecal chemotherapy in adults with BALL, especially in isolated CNSL. More cases and further studies are needed to verify these findings.

Acknowledgements This work was supported by grants from the National Natural Sciences Foundation of China (81400092; to M. Zhao), the Tianjin Key Natural Science Foundation
(17JCZDJC35800, 15JCQNJC45500; to M. Zhao), and the Tianjin Key Science and Technology Program (2015K215, 15KG134, 16KG110; to M. Zhao), as well as Tianjin First Central Hospital. This work was also supported by the Ph.D. Candidate Research Innovation Fund of Nankai University (X. He).

Author contributions MFZ and KQL designed the research; XYH, XX, QL, YYJ, TY, JXM and LM performed the research; XYH, YQC, RS, XJ, WYL and CCL analyzed the data; XYH wrote the manuscript; $\mathrm{XYH}$ and MFZ revised the manuscript. All authors approved the final version of the manuscript.

\section{Compliance with ethical standards}

Ethical approval This study was conducted according to the principles of the Declaration of Helsinki and with the approval of the Ethics Committee of Tianjin First Central Hospital.

Informed consent All the enrolled patients or their families provided written informed consent.

Conflict of interest The authors declare that they have no conflict of interest.

Publisher's note: Springer Nature remains neutral with regard to jurisdictional claims in published maps and institutional affiliations.

Open Access This article is licensed under a Creative Commons Attribution 4.0 International License, which permits use, sharing, adaptation, distribution and reproduction in any medium or format, as long as you give appropriate credit to the original author(s) and the source, provide a link to the Creative Commons license, and indicate if changes were made. The images or other third party material in this article are included in the article's Creative Commons license, unless indicated otherwise in a credit line to the material. If material is not included in the article's Creative Commons license and your intended use is not permitted by statutory regulation or exceeds the permitted use, you will need to obtain permission directly from the copyright holder. To view a copy of this license, visit http://creativecommons. org/licenses/by/4.0/.

\section{References}

1. Maus MV, Grupp SA, Porter DL, June CH. Antibody-modified T cells: CARs take the front seat for hematologic malignancies. Blood. 2014;123:2625-35.

2. Maude SL, Frey N, Shaw PA, Aplenc R, Barrett DM, Bunin NJ, et al. Chimeric antigen receptor $\mathrm{T}$ cells for sustained remissions in leukemia. N Engl J Med. 2014;371:1507-17.

3. Lee DW, Kochenderfer JN, Stetler-Stevenson M, Cui YK, Delbrook C, Feldman SA. et al. T cells expressing CD19 chimeric antigen receptors for acute lymphoblastic leukaemia in children and young adults: a phase 1 dose-escalation trial. Lancet. 2015;385:517-28.

4. Xiao X, He X, Li Q, Zhang H, Meng J, Jiang Y, et al. Plasma exchange can be an alternative therapeutic modality for severe cytokine release syndrome after chimeric antigen receptor-t cell infusion: a case report. Clin Cancer Res. 2019;25:29-34.

5. Dai H, Zhang W, Li X, Han Q, Guo Y, Zhang Y, et al. Tolerance and efficacy of autologous or donor-derived $\mathrm{T}$ cells expressing CD19 chimeric antigen receptors in adult B-ALL with extramedullary leukemia. Oncoimmunology. 2015;4:e1027469.

6. Nellan A, Lee DW. Paving the road ahead for CD19 CAR T-cell therapy. Curr Opin Hematol. 2015;22:516-20. 